\title{
Cadenas de valor. Un enfoque poderoso en la nueva competitividad global
}

\begin{abstract}
René Mendoza $\mathrm{V}^{*}$
* Investigador asociado y profesor visitante de la Universidad Rafael Landívar, Coordinador del Postgrado en Investigación administrado por BICU y URACCAN. E-mail: rmvidaurre@yahoo.com
\end{abstract}

Recibido: agosto 2005 / Aceptado: diciembre 2005

LOS PLANES DE GOBIERNO YA NO SÓLO HABLAN DE CLUSTER, SINO DE CADENAS de valor. Sin embargo, los estudios y cursos universitarios existentes en Nicaragua bajo el título cadenas de valor, en realidad son estudios de cadenas productivas. ¿Qué es pues cadenas de valor? Este artículo pasa revista a los antecedentes de este enfoque para mostrar su evolución y su carácter distintivo, desarrolla la teoría y metodología que hace de la cadena de valor una herramienta poderosa para la competitividad, y concluye revelando sus limitaciones y fortalezas desde países como Nicaragua.

Palabras clave: competencia económica / calidad total / asociaciones empresariales / oferta y demanda / cambio organizacional / precios abiertos

En el Plan Nacional de Desarrollo (2003), el gobierno incluyó el concepto cluster, según M. Porter; el nuevo PND (2005), que resume el viejo PND y la Estrategia Reforzada de Crecimiento y de Reducción de la Pobreza, retiene el concepto de cluster e incorpora el de cadenas de valor. Pero ¿qué es cadenas de valor? Los pocos estudios existentes en el país, unos llevados a cabo por el Ministerio Agropecuario y Forestal (MAGFOR) (1998-2000) y, más recientemente, por el IICA (2004), usan el nombre de cadenas de valor pero, en realidad, se refieren a cadenas productivas, no de valor. En el sector privado del país tampoco se encuentran estudios que apliquen ese enfoque; es en las compañías extranjeras donde se encuentran abundantes estudios. Si se observa el trabajo de las Universidades del país, en el mejor de los casos siguen la tónica del IICA, mientras prestigiosas universidades como London School Economics, Sussex y Harvard University enseñan ese enfoque, considerándolo hoy por hoy, el instrumento más poderoso para expandir, generar, corregir y catalizar competitividad y equidad en el mundo.

¿Qué hace competitiva a una empresa, a una institución o a una comunidad? Antes de continuar, es necesario definir qué es competitividad. Para eso, el mejor maestro es Schumpeter (1942:84): "En la realidad capitalista... no es la competencia por el precio lo que cuenta, sino la competencia sobre el nuevo producto, la nueva tecnología, las nuevas 
fuentes de oferta, el nuevo tipo de organización, una competencia que dirige un costo clave o una calidad y que golpea, no sobre el margen de la ganancia y la producción de la empresa, sino en sus fundamentos y en su vida misma”. En segundo lugar, el enfoque de cadenas sugiere que se compite como empresas encadenadas - no individuales -, explícita o implícitamente coalicionadas. Este artículo parte de ambos puntos, explica el sentido evolutivo de cadenas a través de sus varios enfoques, desarrolla el enfoque de cadenas de valor y ofrece una ponderación sobre su aplicación por cualquier empresa de productos y de servicios.

\section{La noción de cadena en la evolución de los enfoques}

El concepto de cadenas revela la transformación de un producto físico, por ejemplo, el maíz a tortillas pasando por la molienda; posteriormente, se incluye el aspecto técnico en la transformación del producto; después, se visualiza el aspecto físico y su tecnología y se vislumbra también a actores, pueblos y organizaciones; todo ello se enmarca en cadenas globales con poder y gobierno. De esta forma, la noción de cadena productiva adquiere un nuevo sentido, el de valor con significados (Appadurai: "los productos tienen biografía"), emergiendo así la importancia del conocimiento.

Esa evolución del concepto corresponde al de los enfoques (ver cuadro 1). El enfoque inputoutput de W. Leonties (ruso-estadounidense, premio Nóbel 1974) es la transformación del producto físico, utilizado sobre todo en las economías planificadas. Esa idea perdura en el enfoque Filiere, de la escuela francesa, que primeramente desarrolló el enfoque para sus ex - colonias en los años 1960-70, viendo a los productos como un "hilo" donde el rol del mercado es atribuido al Estado. Al mismo tiempo, entre 1970 y 1980 surgió el enfoque Subsector Analysis en la Escuela de Michigan (Boomgard, 1992) que, preocupada por cómo intervenir, hace aparecer a los actores humanos y su organización económica en las cadenas, definiéndose el sub-sector (cadena) como "network of firms that supply raw materials, transform them, and distribute finished goods to a particular consumer market”. Posteriormente, en la década de 1990, aparece Gereffi con el Global Commodity Chains, incluyendo conceptos como poder y gobierno dentro de las cadenas. Finalmente, en el presente milenio, emerge el enfoque cadenas de valor, desarrollado mayormente en la Sussex University por un equipo que previamente trabajó la nueva competitividad en el marco de políticas industriales (R. Kaplinsky, H. Schmitz). Este avance es fortalecido, aunque con muchas críticas, con el actor oriented approach de N. Long de la Waggening University, quien afirma que los significados cambian a lo largo de la cadena y son disputados en cada lugar y tiempo. 
Cuadro 1. Diferencias y contribuciones de cada enfoque

\begin{tabular}{|l|l||}
\hline \hline Escuelas & \multicolumn{1}{|c||}{ Contenido } \\
\hline $\begin{array}{l}\text { Input- } \\
\text { output }\end{array}$ & -Lo físico - país; década de los 1950, Wassily Leonties \\
\hline Filiere & $\begin{array}{l}\text {-Físico y técnico - dimensión internacional, interés en el rol de las empresas de un país } \\
\text { (Francia), y después en sus colonias }\end{array}$ \\
\hline $\begin{array}{l}\text { Análisis } \\
\text { sub- } \\
\text { sectorial }\end{array}$ & $\begin{array}{l}\text {-Varias cadenas } \\
\text {-Preocupación: cómo intervenir, cómo hacer estudios más rápidos, baratos y } \\
\text { apropiados, responder a PYMEs } \\
\text {-Concentrado a producción y procesamiento }\end{array}$ \\
\hline $\begin{array}{l}\text { Global } \\
\text { commodity } \\
\text { Chain }\end{array}$ & $\begin{array}{l}\text {-Rol de gobierno, poder y clasificación de cadenas } \\
\text {-Global; transnacional }\end{array}$ \\
\hline $\begin{array}{l}\text { Cadenas } \\
\text { de valor }\end{array}$ & $\begin{array}{l}\text {-Además de la fase productiva, se añaden nuevas fases: diseño, marketing, reciclaje } \\
\text {-Énfasis en lo intangible } \\
\text {-Pregunta: ¿cómo pueden las pequeñas y medianas empresas insertarse mejor a la } \\
\text { economía global? }\end{array}$ \\
\hline
\end{tabular}

\section{El enfoque cadenas de valor ${ }^{1}$}

Cadena se entiende como la transformación de insumos físicos (Input-output) y de servicios en un producto (Filiere, Subsector analysis), transformación que constituye una fase de varias otras que se ubican tanto hacia atrás (diseño) como hacia delante ("entrega", "venta" y "regreso") y que completan una relación de reciprocidad y funcionamiento sistémico (ver Cuadro 2).

Cuadro 2. Fases de una cadena

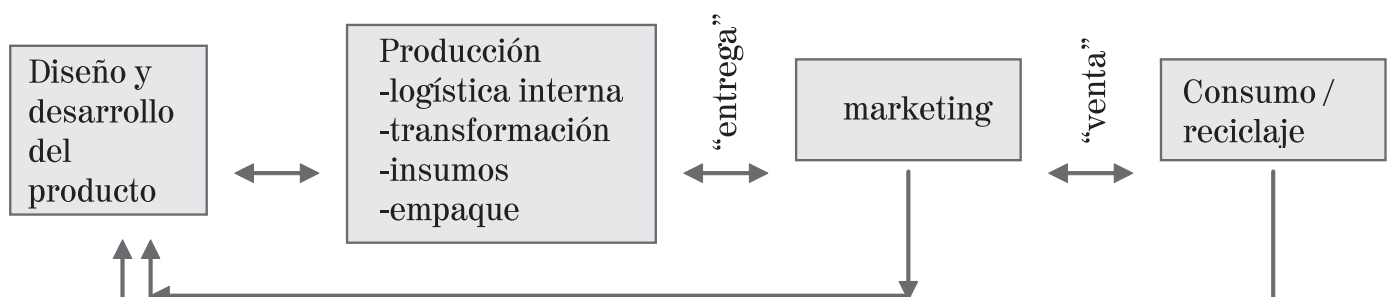

Fuente: basado en Kaplinsky y Morris (2000).

\section{Características del enfoque}

Antes de caracterizar la cadena de valor, es necesario distinguir cuál es la diferencia entre este enfoque y los anteriores. Hasta las cadenas de valor, el concepto de cadenas, en los aspectos del abastecimiento, producción, procesamiento y comercialización se visualizaron sólo física, tecnológica u organizativamente. En el enfoque cadenas de valor, toda esa cadena entra en una sola fase: la producción. Esa fase, además, es considerada como la fase donde menos valor se agrega y donde hay menos renta; es la fase de lo tangible. ¿Dónde 
se crea el valor, entonces? En las otras fases, hacia atrás o hacia adelante.

- Hacia atrás está el diseño de productos, empresas y organización; es la fase donde el ser humano se diferencia de los otros seres porque antes de hacer las cosas, las imagina; esa imaginación es la que crea valor. Por ejemplo, en agricultura, las empresas mundiales más rentables no son los cafetaleros, sino quienes producen semillas y las patentan.

- Hacia adelante, la fase de marketing, de creación de marcas en sus distintas variantes (mark, brand name; marcas ecológicas, marcas de calidad nutricional, marcas de justicia social como el fair trade, certificados de origen), para "vender" toda una vida o una cultura en un concepto (marca) que los clientes hagan suya.

- Hacia más adelante, la fase del reciclaje como indicador de eficiencia y competitividad: quien desaprovecha sus "desechos" es ineficiente y acomodado a viejas prácticas, mientras que las empresas que crean valor desde la "nada" y desde el "estorbo", siguen creando nuevos productos (fase diseño) para satisfacer las infinitas necesidades de la humanidad.

¿Cuáles son las características de las cadenas de valor? Es una cadena extendida verticalmente (modo transformación del producto) y horizontalmente (varios insumos para cada fase o para otras fases). Son varias cadenas originadas en el productor intermediario (ver Cuadro 3). Detrás de la diversidad y extensión de las cadenas, se encuentra lo invisible: el conocimiento y las relaciones que agregan valor a las cadenas.

Cuadro 3. Cadenas de unidades de recurso forestal: Madera-Triángulo Minero-RAAN Nicaragua

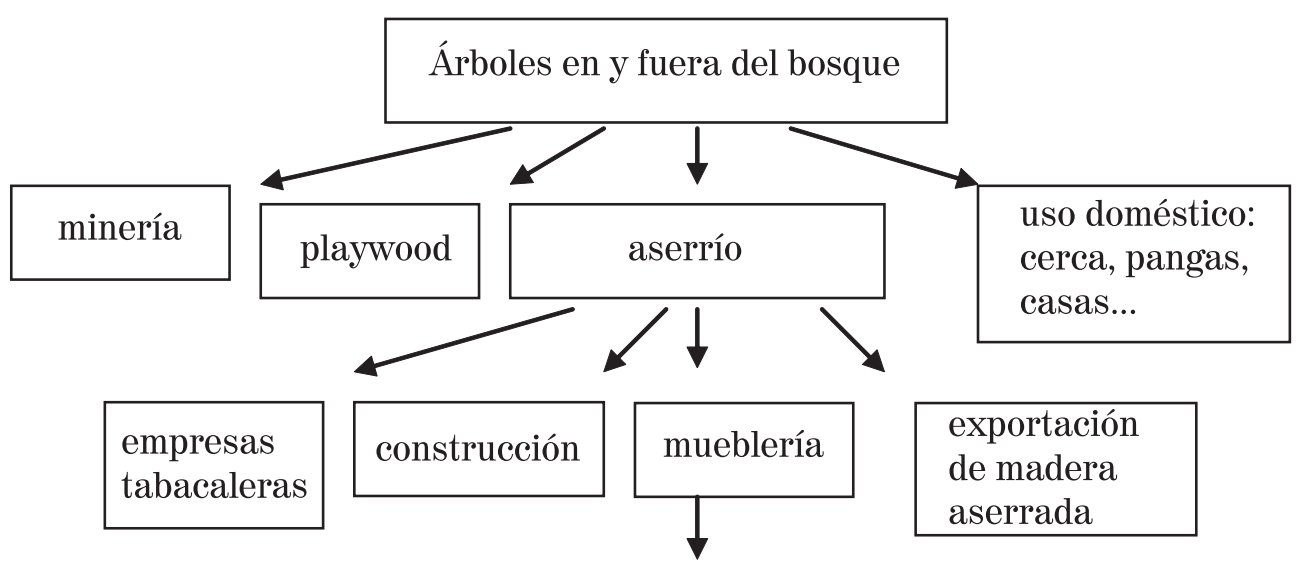

El propósito de los enfoques precedentes es identificar dónde intervenir (Subsector analysis), de parte de las agencias de cooperación; dónde dar asistencia técnica (filiere) de parte del Estado; o cómo formular políticas para la planificación económica (inputoutput). El propósito final del enfoque cadenas de valor, sus características, conceptos y metodologías, es que un conjunto de empresas de distinto tamaño puedan escalar (upgrading). Ver pregunta en el cuadro 1: ¿Cómo pueden hacer las pequeñas empresas para insertarse de mejor manera en la economía global? 


\section{Conceptualización del enfoque}

Tres son los conceptos clave: renta (rent), gobernación (governance) y escalamiento (upgrading). Estos conceptos permiten que las cadenas de valor sean analíticas y superen el carácter descriptivo de los anteriores enfoques. Analizando el concepto renta, en sus perspectivas estática y dinámica, son los ingresos que obtiene cada actor por su capacidad para protegerse de sus competidores, habilidad que se traduce en mejor renta. Para el economista Ricardo, la renta crece con la (desigual) apropiación o control de un recurso escaso, por ejemplo la tierra de parte de terratenientes. Esta noción de Ricardo se ubica en la perspectiva estática, pues la renta sólo depende de controlar determinado recurso escaso. La historia revela que ese "control", normalmente, se ha logrado con mucha violencia. De ahí la noción de acumulación primitiva de Marx, donde lo político-militar domina y la eficiencia no pasa de ser un simple discurso, algo bastante real no sólo para zonas de frontera agrícola, sino para países como Nicaragua.

Por otra parte, según Schumpeter, la escasez puede ser resultado de la innovación que proviene de nuevas combinaciones de factores y de la creación de nuevas condiciones que permiten acceder a una nueva renta que genera una super-ganancia, la cual se disipa cuando los competidores copian las innovaciones para acceder a la nueva renta; es el momento en que se genera un ambiente de búsqueda de mayores y nuevas rentas en sus variadas formas. Esta noción de renta se ubica en la perspectiva dinámica, porque las nuevas rentas se van añadiendo en el tiempo, y las rentas viejas se van erosionando mediante las fuerzas de la competencia. Por ejemplo, en la producción de zapatos y textiles ya no hay secretos: la nueva renta está en el diseño y en las redes comerciales; en la producción de automóviles, la renta descansa en la investigación; o rentas para productores protegidos por las políticas de un gobierno. Por ejemplo, el azúcar en Nicaragua. De donde se deduce que hay diversos tipos de renta que, en cuanto se refieren a la renta económica, se resumen en el Cuadro 4. Estas rentas son, básicamente, la diferencia en productividad de los factores y las barreras de entrada, y pueden clasificarse como endógeno o exógeno a la empresa y a las cadenas.

Cuadro 4. Tipos de renta económica

\begin{tabular}{|c|c|c|}
\hline \multicolumn{2}{|r|}{ Renta económica } & Concepto \\
\hline \multirow{5}{*}{ 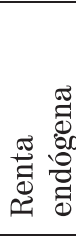 } & Renta tecnológica & Control sobre tecnología escasa \\
\hline & $\begin{array}{l}\text { Renta en recursos } \\
\text { humanos }\end{array}$ & Acceso a mejores habilidades que la competencia \\
\hline & Renta organizacional & Formas superiores de organización interna \\
\hline & Renta marketing & Mejores capacidades de marketing y marcas valiosas \\
\hline & Renta relacional & Calidad de relaciones superior con los proveedores y clientes \\
\hline \multirow{4}{*}{ 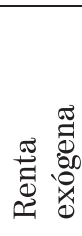 } & Renta de recursos & Acceso a escasos recursos naturales \\
\hline & Renta de políticas & $\begin{array}{l}\text { Operar con gobierno eficiente, construyendo barreras para la } \\
\text { entrada de competidores }\end{array}$ \\
\hline & Renta infraestructura & $\begin{array}{lccccc}\begin{array}{l}\text { Acceso a alta } \\
\text { telecomunicaciones }\end{array} & \text { calidad en infraestructura } & \text { como }\end{array}$ \\
\hline & Renta financiera & Acceso a crédito en mejores términos que la competencia \\
\hline
\end{tabular}


El segundo concepto clave es el de gobernación, que parte de dos premisas. Primera: por mucha dispersión que haya entre las empresas, lejos de estar coordinadas por "la mano invisible", hay coordinación y vínculos en toda actividad y sistemas de producción; segunda: la coordinación no es equivalencia de gobierno (líder). Ejercer gobierno no es sólo coordinar; ni captar la mayor renta convierte automáticamente a una empresa en gobierno ó líder. El gobierno de una cadena identifica a las empresas y a los actores clave en y afuera de la cadena y se responsabiliza en ayudarlos a escalar pues, de lo contrario, toda la cadena será menos competitiva; el gobierno desarrolla un horizonte sobre la base de su posicionamiento, logística, integración de componente en el diseño de productos finales, monitoreo de resultados, establecimiento de relaciones entre varios actores, organización de la oferta y la provisión de insumos. En ese rol beligerante del gobierno, se puede ser despótico o democrático, dependiendo de la transparencia con que se fijen las reglas y se cumplan. En la medida en que un gobierno se compenetre con el conjunto de la cadena, gozará o no de legitimidad ante el resto de las empresas de la cadena.

La diferencia de gobiernos no sólo se debe a su despotismo o a la construcción de partnership (socios) a lo largo de la cadena, sino que hay separación de poderes y tipos de gobierno en y fuera de la cadena. Sobre la separación de poderes, ver Cuadro 5 donde el enfoque, prestando conceptos a las ciencias políticas, distingue lo que corresponde a cada área del gobierno (ejecutivo, legislativo y judicial), y aplicando esta noción de gobierno y renta, resulta el Cuadro 6, donde se expresan dos tipos de cadenas de valor desde donde se crean rentas. Por ejemplo, renta en diseño desde el tipo buyer driven, o renta en investigación desde el producer driven.

Cuadro 5. Roles de los poderes

\begin{tabular}{|l|l|l|}
\hline $\begin{array}{l}\text { Poderes en la } \\
\text { cadena }\end{array}$ & $\begin{array}{l}\text { Ejercidos por miembros de una } \\
\text { cadena }\end{array}$ & $\begin{array}{l}\text { Ejercidos por actores externos a } \\
\text { la cadena }\end{array}$ \\
\hline Legislativo & $\begin{array}{l}\text {-Fijar estándares para proveedores } \\
\text { sobre entrega, frecuencia y calidad } \\
\text { de insumos. }\end{array}$ & $\begin{array}{l}\text {-Estándares ambientales, trabajo } \\
\text { infantil } \\
\text {-ISO 9000, 14000, SA8000, HACCPP }\end{array}$ \\
\hline Judicial & $\begin{array}{l}\text {-Monitoreo del avance de los } \\
\text { proveedores según los estándares } \\
\text { acordados }\end{array}$ & $\begin{array}{l}\text {-Monitoreo estándar, trabajo } \\
\text { infantil, avance ISO. }\end{array}$ \\
\hline Ejecutivo & $\begin{array}{l}\text {-Asistencia a proveedores a cumplir } \\
\text { con los estándares } \\
\text {-Asociaciones de productores que } \\
\text { ayudan a sus miembros lograr } \\
\text { estándares }\end{array}$ & $\begin{array}{l}\text {-Servicios especializados: Asesoría } \\
\text {-Política indeligencia de mercado } \\
\text { parte del gobierno de apoyo de }\end{array}$ \\
\hline
\end{tabular}


Cuadro 6. Tipos de gobierno

\begin{tabular}{|l|l|l|}
\hline & Producer driven & Buyer driven \\
\hline Mando en cadena & Capital industrial & Capital comercial \\
\hline Competencia central & R \& D; producción & Diseño, marketing \\
\hline Barreras entrada & Escala & Dimensión \\
\hline Industrias típicas & Carros, computadoras & Juguetes, ropa \\
\hline Principal vínculo & Base en inversión & Base en comercio \\
\hline $\begin{array}{l}\text { Estructura relación } \\
\text { predominante }\end{array}$ & Vertical & Horizontal \\
\hline
\end{tabular}

En esencia, un gobierno promueve que sus contrapartes escalen (upgrading), proceso para el cual busca deliberadamente pasar de productos tangibles a intangibles, como el diseño o el marketing, y construir una visión (horizonte) para que sus distintos aliados en una cadena, que están compitiendo como cadena, puedan ver más allá de su propio negocio y cada vez más con productos intangibles (organización, relaciones sociales, conocimiento). Esa es la alternativa de gobierno que se va creando: pasar de lo tangible a lo intangible.

Finalmente, el concepto de escalamiento (upgrading). El acceso a una renta mayor es resultado del gobierno (de la cadena, no gobierno de un país) y de la calidad de sus relaciones con sus contrapartes, de donde surge la innovación cuando los resultados son mayores que la competencia, momento en el que se habla de escalamiento (upgrading). Éste, a su vez, reconoce sus dotaciones relativas y por lo tanto la existencia de la renta. Pero, ¿́cómo identificar ese escalamiento? Hay varias teorías, dos de las cuales se expresan en el Cuadro 7 , que denotan que es la capacidad de las empresas encadenadas la que genera cadenas de valor creando escasez y renta de forma permanente. Obsérvese que ambas escuelas están dirigidas a empresas individuales, pero desde el enfoque de cadenas de valor, las dos escuelas son captadas para trabajar innovaciones en cadenas, con un énfasis no al interior de una empresa, sino entre empresas, entre fases, entre empresas de productos y de servicios. 
Cuadro 7. Teorías aprovechadas por el enfoque cadenas de valor

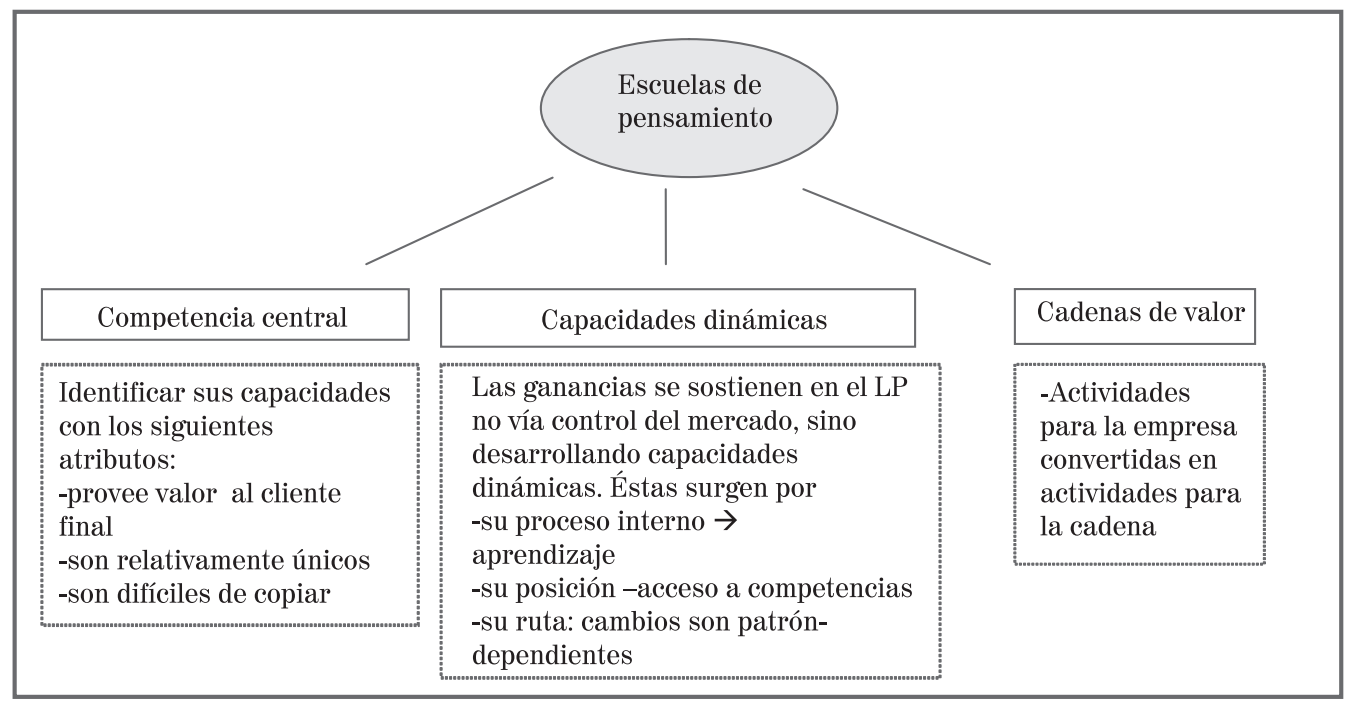

Se puede afirmar que competitividad es la capacidad permanente de aprender, lo que resulta en innovación permanente y que se traduce en tipos de escalamiento que presentamos en el Cuadro 8. De estos, la mayor renta está en las actividades intangibles: el escalamiento en producto es lo que se puede ver (producto visible); el de proceso lo invisible (intangible); el de funciones es la entrada a nuevas fases y abandono de otras. Por ejemplo, dejar de hacer queso y saltar a su distribución - y el de escalamiento en cadenas que es dejar de hacer un producto y saltar a - ó desarrollar - otro producto (contenido cualitativo, empaque, imagenconcepto).

Cuadro 8. Tipos de escalamiento

\begin{tabular}{|l|l|l|}
\hline Tipo & Dentro fases o empresas tecnología, & Entre fases o empresas \\
\hline Proceso & $\begin{array}{l}\text {-Introducción nueva } \\
\text { eficiencia interna } \\
\text {--Menos bodegaje, desecho }\end{array}$ & $\begin{array}{l}\text {-Rádez de entrega de productos y } \\
\text { cantidades menores }\end{array}$ \\
\hline Producto & $\begin{array}{l}\text {-Introducción nuevos productos o } \\
\text { mejoría de procedimientos. Por ejemplo, } \\
\text { arroz orgánico, nuevo empaque. }\end{array}$ & $\begin{array}{l}\text {-Introducción nuevos productos o } \\
\text { mejoría de la calidad del producto }\end{array}$ \\
\hline Funciones & $\begin{array}{l}\text {-Nueva función o abandono de otra } \\
\text { función } \\
\text {-Asumir/dejar control calidad }\end{array}$ & -De hacer queso a distribuirlo \\
\hline Cadena & $\begin{array}{l}\text {-Captando nueva función, se escala a } \\
\text { otra cadena. }\end{array}$ & -De trillar arroz a hacer pinol \\
\hline
\end{tabular}


Gobernar para escalar y así acceder a nuevas y nuevas rentas, renta como resultado de una acción de gobierno en un proceso de escalamiento. Renta-gobierno-escalamiento expresa un triángulo conceptual para analizar cadenas de valor. Sin el uso de ese triángulo conceptual, los estudiosos se quedan en cadenas productivas. En adición, el uso de ese triángulo conceptual implica reconocer que la base de la competitividad es el conocimiento, que se genera en un marco de relaciones. Ahí está el reto: quien aprende más rápido es más competitivo; en este caso, no importa si una empresa aprende o no, sino que el conjunto de empresas encadenadas van estableciendo mecanismos de aprendizaje permanente ("lo perfecto es enemigo de la mejora”) y, por lo tanto, es importante invertir en relaciones sociales. Por ejemplo, renta relacional - para generar más y nuevas rentas.

\section{Importancia, aplicación y limitaciones}

Hasta aquí se ha expuesto la utilidad y aplicación de las cadenas de valor. Para ponderarlas globalmente, es necesario desglosar más estos aspectos.

\section{Importancia}

La importancia de las cadenas de valor radica en cuatro razones. Primera, la competencia sistémica es clave en la creciente división del trabajo y la dispersión global de los componentes de producción. En términos históricos, la contribución de Smith (perspectiva desde la empresa-mercado) fue expandir el mercado para una mayor división del trabajo que permitiría mayor especialización y mecanización de la producción; igualmente, la de Taylor (perspectiva desde la planta de producción) fue que, a mayor escala, mayor número de estaciones y aumento de eficiencia en el trabajo de cada estación a través del manejo científico; la competencia sistémica, por su parte, constituye un salto de la perspectiva intra-planta e intra-empresa hacia un enfoque sistémico:

- Just in time vislumbra la existencia de "islas de eficiencia", planteando reducir los stock de productos (bodega) para ganar eficiencia en el conjunto del sistema;

- La rapidez de reacción ("paralelo/concurrente") de las distintas fases, necesita de puentes entre las fases. Desde esta perspectiva, se retoman los enfoques de core competence y outsourcing: concentrarse en algo único, difícil de ser copiado en los servicios que se provee, y "expulsar" funciones donde se es menos competente, pero donde otras empresas sí lo son, precisamente para garantizar competencia sistémica (ver enfoque de Moore sobre Ecosistemas de Negocios).

Segunda, ir más allá de la eficiencia de la producción. El análisis de cadenas de valor ayuda a entender cómo los productores insertados en el mercado final pueden influir en su habilidad de conseguir mejores ganancias a través de su participación. ¿Cómo?

- Conociendo la lógica de inserción de las transnacionales en nuestras economías. Esas empresas ubican subsidiarias en los países del sur, debido a los bajos costos de trabajo y materia prima; a las políticas comerciales en el ámbito internacional (tarifas, cuotas, acuerdos por pertenecer a una región); y por otros criterios estratégicos como el aprovechamiento de las raíces étnicas, o proveerse de determinado insumo demandado 
en la competencia global. Estos conocimientos tienen el objetivo de conectar las empresas de la cadena que interesa a la ruta de las transnacionales para obtener su modernidad y para que se enraícen en la economía nacional.

- Reconociendo el potencial competitivo de las empresas nacionales. Muchas empresas nacionales, con productos (y sub-productos) tradicionales, han sido competitivas por décadas. El reto es ponderar toda la cadena y su potencial de expandir el mercado.

El enfoque cadenas de valor permite discernir los factores que determinan la participación de un grupo en el mercado final; identificar las ventajas y desventajas de empresas y países especializados en producción en lugar de servicios; descubrir las formas particulares de conexión que afectan el escalonamiento de las empresas. Por ejemplo, las transnacionales suelen apoyar a los pequeños a mejorar su producción, pero impiden que accedan al diseño o a otros mercados.

Tercera, el enfoque cadenas de valor permite explicar la distribución de los beneficios, especialmente ingresos. La globalización es una constante caída de barreras al flujo global de información, ideas, factores de producción (especialmente capital y trabajadores capacitados), tecnologías y productos. Es una dinámica con ganadores y perdedores en el norte y en el sur, entre trabajadores capacitados y no capacitados, en una región y dentro de un país. De este modo, se identifican dos rutas: la ruta cuesta arriba y la ruta cuesta abajo. La segunda se produce cuando se aumenta la producción, pero el valor baja (ruta de la miseria); la primera es cuando la participación creciente y mejorada en la economía global permite un crecimiento sostenible de los ingresos. Por lo tanto, la clave no es tanto participar o no en la globalización, sino cómo hacerlo de un modo que permita un crecimiento sostenible. Para ello, se estudia la naturaleza y determinantes de la competitividad (individual = $>$ grupal), y las actividades donde se logran retornos crecientes.

Finalmente, cadenas de valor entra en contradicción con la noción de desarrollo basado en una perspectiva territorial - desarrollo del Estado-nación o desarrollo nacional, noción superada porque no puede explicar el mundo global de hoy. Cadenas de valor va en concordancia con la noción de desarrollo concebido desde lo social, redefiniendo el triángulo espacio/grupos sociales/desarrollo en un marco transnacional. En consecuencia, hay grupos desarrollados (y sub-desarrollados) en el norte y en el sur, barrios residenciales apartheid en Managua, Sao Paulo y en New York; igualmente, hay asentamientos y fabelas más allá de la dualidad norte-sur. La competitividad ya no se da entre empresas individuales, sino entre cadenas globales y coaliciones humanas que trascienden los territorios que son una construcción social, y no una línea administrativa. Una empresa saca un producto pensando ubicarlo, no en un país, sino en un sector social ubicado en cualquier parte del globo terrestre; también Nicaragua puede llevar las rosquillas detrás de los nicas emigrantes, y no sólo las rosquillas, sino también las costumbres y, sobre todo, el conocimiento².

\section{Aplicación del enfoque y la emergencia de los dilemas}

Muchos conceptos y roles institucionales son repensados desde el enfoque cadenas de valor. Con este enfoque, es posible preguntarse, en tanto profesional, institución de desarrollo, empresa creadora de empleos o centro de investigación: ¿Dónde estoy? ¿Cuando llegar a 
las cadenas de valor? La hipótesis de este trabajo es que la mayoría de las instituciones y empresas (de productos y servicios) se han quedado en el nivel del input-output ó, a lo sumo, en el de Filiere. Con esto, la pregunta se hace extensiva a la ubicación en el ámbito de lo tangible (producto y servicios) o de lo intangible (relaciones) recreando nuevos y nuevos productos. En consecuencia, el enfoque ayuda a repensar los roles: resulta que la mayoría de las ONGs y empresas están en lo tangible, en una sola fase y, dentro de esa fase de producción, sólo están en el primer sub-nivel. Por ejemplo, quienes trabajan en desarrollo rural (al igual que la mayoría de las consultorías) sólo están en producción (finca), no donde se crea más valor. Este es un “árbol” que el enfoque sacude con placer.

Otro “árbol” se refiere a género. Desde el enfoque cadenas de valor, se ve a las mujeres como protagonistas (empresarias) y no sólo como víctimas hacia las que sentir compasión, como sugieren los enfoques tradicionales de género ${ }^{3}$. Mapeando la diversidad de subproductos de casi cualquier rubro, se encuentra la presencia masiva de las mujeres con sus habilidades, conocimientos, redes sociales y visión. Por mencionar sólo un ejemplo, el producto maíz tiene más de 20 subproductos, uno de los cuales es la tortilla, que es una invención de la cultura maya. No se hace tortilla en África ni en América del Sur. Es decir, hacer tortilla no sólo requiere habilidades, sino que expresa toda una cultura milenaria. La competitividad está tanto en reconocer a las mujeres en fases de alto valor, sino en tomarlas en cuenta seriamente como oportunidad para distinguir a la cadena en su conjunto y graduarla como cadena "de valor".

El tercer "árbol” es el conocimiento. El enfoque cadenas de valor anima a explicar cómo una empresa es competitiva, no tanto si gana o pierde. ¿Qué la hace competitiva? ¿Qué agrega valor a una cadena? Puede ser una red social, pero ¿de qué tipo? ¿Cómo es ese factor y cómo se mide económicamente? Es decir, ¿cómo se mide la innovación? ¿Qué produce cadenas de valor exitosas o fracasadas?

La hipótesis de este trabajo apunta a que no es la infraestructura básica ligada a un espacio físico la que genera cadenas de valor, sino la diversidad social (estructura y capital social, saber local) lo que define el espacio físico y determina vía "vínculos" a las cadenas de valor; según esto: ¿Cómo se vincula una familia, la mujer o una organización social con las cadenas? ¿Cómo se vincula la cultura (construcción social) con la cadena de valor? Responder a estas preguntas es tener la "llave" para ser competitivo como cadenas y como sociedades.

Finalmente, el último "árbol” se relaciona con marketing y mercado. Mercado, según los indígenas Incas de Sur América, es un lugar de encuentro y fiesta; para los mestizos, es un lugar de explotación y tristeza. El enfoque cadenas de valor ayuda a que sea lugar de fiesta: el Jordán es más que el Jordán, el queso es más que la leche, el corte es más que el hilo, el maduro frito es más que el plátano. Sin embargo, la lógica del department store revela que la fantasía sin vida (comercial) se impone en los productos, de tal manera que se compra determinado "tabaco" para ser como la "bailarina". En consecuencia, muchas personas acuden y, aunque no compren, tienen fantasías con la bailarina. La mercancía siempre fue más que mercancía. La clave está en que hay que relacionarse con el corte, no separar corte-hilo-vida humana. El enfoque permite concebir que no se trata solo de la cultura, de 
la relación social, sino del cambio de la fantasía. El hombre ve a la "mujer perfecta" y su esposa no logra ser su agencia; o la mujer ve al "hombre perfecto" y su esposo no logra ser su agencia. Marketing es algo concreto, presentación de fantasías: Nestlé presenta una fantasía que tiene agencia: por ejemplo, en la botella de agua. Cadenas de valor ofrece otra forma de hacer fantasía, de construir marcas donde la "esposa" o el "esposo" (culturas, sociedades) sean agencia.

\section{Limitaciones}

Valorando el conjunto, una limitación estructural del enfoque es que asume la economía global como algo dado, sin problematizarla, hablando sólo de "insertarse" lo que, aplicado al mundo rural, ha significado que las familias campesinas se conviertan en mano de obra barata. ¿Cómo desarrollar subcontracting entre empresas grandes y familias campesinas? ¿Cómo pueden escalar las familias campesinas como cadena sobre la base de su mercancía? Otra limitación es que no se cuestiona que las cadenas de valor son verdaderas instituciones que no dejan entrar a otros actores, mucho menos a los más pequeños.

Las limitaciones, sin embargo, son una ventana para mejorar el enfoque sobre la base de otros enfoques, como el enfoque etnográfico; la Nueva Economía Institucional con los costos de transacción y el dilema de la acción colectiva; la teoría del capital social; la creación de futuro, etc.

\section{Notas}

1. Esta sección está basada fundamentalmente en autores como Kaplinsky y Schmitz, cuyos trabajos son los más completos para el enfoque cadenas de valor.

2. Ver Mendoza y Kuhnekath, 2004, “Desarrollo ¿de quién? ¿Para quiénes?” En: Revista Confidencial, $2004: 391$ http://www. confidencial.com.ni/columnista1-391.htm

3. La preocupación por la perspectiva de género ha llevado a los proyectos y programas de desarrollo a incluir a las mujeres en la producción agropecuaria, porque eso supone que "van a ser valoradas". Pero estas medidas son más bien contraproducentes, pues las mujeres siguen realizando sus trabajos domésticos y, con su inclusión en la producción, realizan doble jornal, gracias a la "perspectiva de género" de las ONGs. Es decir, se cae en la peor trampa: buscando equidad, se genera mayor inequidad.

\section{Referencias bibliográficas}

-ARTOLA, N. y PARRILLI, D. (2003). El Despegue del cluster de productos lácteos de Boaco y Chontales, BID-FOMIN Trust Fund Italiano, Nitlapan-UCA, Managua -BARRIENTOS y WARE BARRIENTOS, (2002). Extending Social protection to informal workers in the horticulture global value chain FALTAN DATOS

-BOOMGARD, J. (1992). "A Subsector Approach to Small Enterprise Promotion and Research" . World Development, vol. 20.2 DONDE Y QUIEN EDITA

-FITTER y KAPLINSKY (2001). Can an agricultural 'commodity' be de-commodified, and if so, who is to gain? DONDE Y QUIEN EDITA

-KAPLINSKY y MORRIS. A Handbook for Value Chain Research. http:/www.ids.ac.uk/ids/ global/valchn.html FALTAN DATOS. Ajustar a normativa. 
-LONG, N. y VILLAREAL, M.. (1998). "Small Product, Big Issues: Value Contestations and Cultural Identities in Cross-border Commodity Networks". Development and Change, Vol., 29 DONDE Y QUIEN EDITA

-MCCORMICK y SCHMITZ, Manual for value chain research on homeworkers in the garment industry FALTA EL AÑO, EL LUGAR Y LA EDITORIAL

-MENDOZA, R. y KUHNEKATH, K. (2004). Tratados de Libre Comercio, PND y Clusters ¿Cambio de Ruta o más de lo Mismo? . Encuentro, No. 67, Managua.

-MENDOZA, R. (2004). "Hacia una segunda generación del comercio justo. Joint Venture-Consorcios, aprendizaje horizontal e investigación participativa universitaria”AQUI FALTA TODO

-MENDOZA, R., La paradoja del café, Nitlapan-UCA, Managua FALTA EL AÑO

-MENDOZA, R. y KUHNEKATH, K., (2004). “Desarrollo ¿de Quién? ¿Para Quiénes?”. Revista Confidencial, 2004: 391.

-PELUPESSY, W., El enfoque de la cadena global de mercancías como herramienta analítica en las economías en desarrollo. http//ivo.uvt.nl/people/pelupessy.spn FALTA EL AÑO

-RAIKES, P., FRIIS, M., y PONTE, S. (2000). Global Commodity Chain analysis and the French Filiere Approach: comparison and critique. Center for Development Research. No.3 FALTA EL LUGAR DE EDICION

-RODRIK, D., (2003). Economic development as self-discovery en:_http://ksghome.harvard. edu/ .drodrik.academic.ksg/papers.html AJUSTAR A LAS NORMAS editoriales

SCHUMPETER, J. (1942). Capitalism, Socialism and Democracy, Hasper and Brothers, New York. EDITORIAL 